\title{
Geometric Error Estimation and Compensation in Compact Spherical Loudspeaker Array Calibration
}

\author{
Franz Zotter \\ Institute of Electronic Music and Acoustics \\ University of Music and Performing Arts \\ Inffeldgasse 10/3, $8010 \mathrm{Graz}$ \\ Email: zotter@iem.at
}

\author{
Balázs Bank \\ Dep. of Measurements and Information Systems \\ Budapest University of Technology and Economics \\ Magyar tudósok körútja 2, H-1117 Budapest \\ Email: bank@mit.bme.hu
}

\begin{abstract}
Compact spherical loudspeaker arrays are nowadays investigated as sources for directional measurements and audio playback. Their microphone-based calibration is not trivial as it requires an elaborate measurement setup of highly accurate geometry. This contribution shows how measurements of accurate geometric alignment were obtained to the authors' best knowledge, and systematically discusses how possible inaccuracies affect the resulting spherical harmonic radiation patterns. Eventually, a method is developed to estimate geometric errors from the measured responses. This geometry estimation is verified by tests with intentional errors. Finally, results are successfully employed to make calibration measurements more robust against geometric errors of the setup. This is done by both time realignment of the responses compensating for radial errors and the correction of the angles in the spherical harmonic transform.
\end{abstract}

\section{INTRODUCTION}

Dodecahedral loudspeakers are common measurement tools in room acoustics due to their nearly omnidirectional sound radiation. The idea behind these arrays was to have a standardized source for measuring room acoustic parameters. As, however, a human speaker or musical instrument do not radiate omnidirectionally, researchers have been considering acoustic sources with variable radiation patterns adjusted to fit any target, [1], [2], [3], [4]. This was shown to improve the accuracy of quality measures for speech intelligibility or the clarity of music reproduction, cf. [5].

The radiation and control of compact spherical loudspeaker arrays is usually described in terms of spherical harmonics, which form basis functions similar to that of the Fourier series, but depend on the zenith $\vartheta$ and azimuth $\varphi$ angles. They appear in the solution of the Helmholtz equation of spherical boundary value problems and therefore provide welldescribed means for acoustic holophony, the synthesis of controlled sound radiation [6]. The sound pressure of any acoustic radiator with limited order $n \leq \mathrm{N}$ is

$$
p(k r, \boldsymbol{\theta})=\sum_{n=0}^{\mathrm{N}} \sum_{m=-n}^{n} \psi_{n m} \frac{h_{n}(k r)}{h_{n}\left(k \mathrm{r}_{\mathrm{M}}\right)} Y_{n}^{m}(\boldsymbol{\theta}),
$$

wherein $\boldsymbol{\theta}(\varphi, \vartheta)=\left[\begin{array}{lll}\cos \varphi \sin \vartheta, & \sin \varphi \sin \vartheta, & \cos \vartheta\end{array}\right]^{\mathrm{T}}$ is a normalized direction vector depending on the zenith $\vartheta$ and azimuth $\varphi, k$ is the wave number, $n$ and $m$ are order and degree of the spherical harmonic $Y_{n}^{m}(\boldsymbol{\theta})$, respectively, $h_{n}(k r)$ is the spherical Hankel function of the second kind, $\psi_{n m}$ are the spherical harmonic coefficients describing the directivity pattern at the radius $r_{M}$, and $i^{2}=-1$; cf. [7]. At the ideal radius $r=\mathrm{r}_{\mathrm{M}}$, the radiation produced remains to be a spherical harmonics expansion

$$
p(\boldsymbol{\theta})=\sum_{n=0}^{\mathrm{N}} \sum_{m=-n}^{n} \psi_{n m} Y_{n}^{m}(\boldsymbol{\theta})
$$

which is determined by the spherical spectral coefficients $\psi_{n m}$.

Radiation synthesis aims to produce arbitrary radiation as specified by Eq. (2). Calibration is done by measuring the directivity patterns $p^{(l)}\left(\boldsymbol{\theta}_{\text {mic }}^{(i)}\right)$ of all $l=1, \ldots$, L loudspeakers with $i=1 \ldots \mathrm{M}$ microphones at uniformly surrounding directions $\theta_{\text {mic }}^{(i)}$ on a constant radius $r_{M}$. The spherical harmonic coefficients $\psi_{n m}^{(l)}$ are obtained by the least squares solution of M simultaneous Eqs. (2) [8], i.e.

$$
\boldsymbol{p}^{(l)}=\boldsymbol{Y}_{\mathrm{mic}} \boldsymbol{\psi}^{(l)} \Longrightarrow \boldsymbol{\psi}^{(l)}=\left(\boldsymbol{Y}_{\mathrm{mic}}^{\mathrm{T}} \boldsymbol{Y}_{\mathrm{mic}}\right)^{-1} \boldsymbol{Y}_{\mathrm{mic}}^{\mathrm{T}} \boldsymbol{p}^{(l)}
$$

using the vectors $\boldsymbol{p}^{(l)}:=\left[p^{(l)}\left(\boldsymbol{\theta}_{\text {mic }}^{(i)}\right)\right]_{i}$ and $\boldsymbol{\psi}^{(l)}:=\left[\psi_{n m}^{(l)}\right]_{n m}$ and the matrix $\boldsymbol{Y}_{\text {mic }}=\left[Y_{n}^{m}\left(\boldsymbol{\theta}_{\text {mic }}^{(i)}\right)\right]_{i}^{n m}$.

The task in radiation control is to design a frequencydependent matrix $\boldsymbol{G}(\omega)$ of which each column contains complex "gains" to produce one spherical harmonic [8] by superimposing the loudspeakers' directivities $\Psi(\omega)=$ $\left[\boldsymbol{\psi}^{(1)}(\omega), \ldots, \boldsymbol{\psi}^{(\mathrm{L})}(\omega)\right]$ so that

$$
\boldsymbol{\Psi}(\omega) \boldsymbol{G}(\omega)=\boldsymbol{I} .
$$

A desired spherical directivity $\psi$ is synthesized by the gains $G(\omega) \psi$.

Driving the array through a decoder $\boldsymbol{D}_{\mathrm{ls}}=\boldsymbol{Y}_{\mathrm{ls}}\left(\boldsymbol{Y}_{\mathrm{ls}}^{\mathrm{T}} \boldsymbol{Y}_{\mathrm{ls}}\right)^{-1}$ is advantageous and yields a tentative, frequency independent control. A modified control system $\tilde{\boldsymbol{G}}(\omega)$ is then employed to perfect the rough synthesis of $\boldsymbol{D}_{\mathrm{ls}}$

$$
\boldsymbol{\Psi}(\omega) \boldsymbol{D}_{\mathrm{ls}} \tilde{\boldsymbol{G}}(\omega)=\boldsymbol{I} .
$$

This formulation usually yields a sparse matrix $\tilde{\boldsymbol{G}}(\omega)$, whose implementation is more efficient than the one of $\boldsymbol{G}(\omega)$, cf. [9]. Its simplified role after the rough synthesis using $D_{\mathrm{ls}}$ is to accomplish frequency independence of all synthesized spherical harmonics and to remove all spherical harmonics that are 
erroneously synthesized due to acoustic crosstalk. A synthesis of $\psi$ is obtained by the gains $D_{\mathrm{ls}} \tilde{G}(\omega) \psi$.

It is clear that when the microphone-based directivity calibration is inaccurate, then the control system $\tilde{\boldsymbol{G}}(\omega)$ will try to produce correct patterns using wrong data. This not only brings additional complexity into the control system and counteracts efficient implementations but also destroys radial directivity focusing. Hence accurate measurements are paramount in the design of the control system. The most important source of errors is geometric: loudspeaker array or microphones that are misplaced by just a few centimeters can render measurement data useless for control.

The present paper focuses on such geometric errors, their estimation, and the minimization of their impact. After describing a real measurement scenario and possible geometric deficiencies, the effects of geometric errors are systematically discussed. Then, the acoustic delay from a spherical loudspeaker array to a measurement point is modeled acoustically, and shown to produce the results of a geometric model after minimum phase removal. Based on this model, we present, to our knowledge, the first method for estimating such measurement geometry errors. The estimates are applicable to check the accuracy of the geometry and to successfully diminish geometry-induced errors prior to radiation control design.

\section{MEASUREMENT OF SPHERICAL LOUDSPEAKER ARRAYS}

We have performed impulse response measurements on the available arrays of IEM, one for the low- (with radius $\mathrm{R}=$ $28.5 \mathrm{~cm}$ using $\mathrm{L}=20$ loudspeaker elements, Fig. 1(a)) and the other one designed for the mid-frequency range $(\mathrm{R}=8.5 \mathrm{~cm}$, $\mathrm{L}=16$, Fig. 1(b)).

Each loudspeaker array was mounted on a turntable, while the radiated measurement signal was recorded with a semicircular arc of 18 microphones at a radius $r=0.7 \mathrm{~m}$ covering all zenith angles in $10^{\circ}$ steps. The turntable permits repeated measurement in $10^{\circ}$ azimuthal steps in order to sequentially gather impulse responses measured by a virtual spherical microphone array with $18 \times 36=648$ positions in zenith and azimuth in total. Ideal positions $\mathbf{X}_{\text {mic }}$ of the virtual array are defined by sequentially applying rotations $\boldsymbol{R}_{\mathrm{z}}$ around $z$ on the coordinates $\mathbf{X}_{\text {arc }}$ of the semicircle

$$
\begin{aligned}
\mathbf{X}_{\mathrm{mic}} & =\left[\boldsymbol{R}_{\mathrm{z}}\left(0^{\circ}\right) \mathbf{X}_{\mathrm{arc}}, \boldsymbol{R}_{\mathrm{z}}\left(10^{\circ}\right) \mathbf{X}_{\mathrm{arc}}, \ldots, \boldsymbol{R}_{\mathrm{z}}\left(350^{\circ}\right) \mathbf{X}_{\mathrm{arc}}\right] \\
\mathbf{X}_{\mathrm{arc}} & =0.7 \mathrm{~m}\left[\begin{array}{cccc}
\cos \left(5^{\circ}\right) & \cos \left(15^{\circ}\right) & \ldots & \cos \left(175^{\circ}\right) \\
0 & 0 & \ldots & 0 \\
\sin \left(5^{\circ}\right) & \sin \left(15^{\circ}\right) & \ldots & \sin \left(175^{\circ}\right)
\end{array}\right], \\
\boldsymbol{R}_{\mathrm{z}}(\varphi) & :=\left[\begin{array}{ccc}
\cos (\varphi) & -\sin (\varphi) & 0 \\
\sin (\varphi) & \cos (\varphi) & 0 \\
0 & 0 & 1
\end{array}\right] .
\end{aligned}
$$

\section{A. Geometric errors}

The array geometries of the semicircular array and the loudspeaker arrays and the incremental rotation of the turntable are highly accurate compared to errors appearing in the relative positioning of both arrays. Generally, a vector $\boldsymbol{x}$ transforms to

$$
\boldsymbol{x}^{\prime}=\boldsymbol{R}(\boldsymbol{r}) \boldsymbol{x}+\boldsymbol{d},
$$

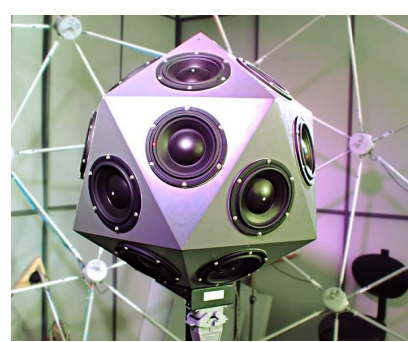

(a) low-frequency array

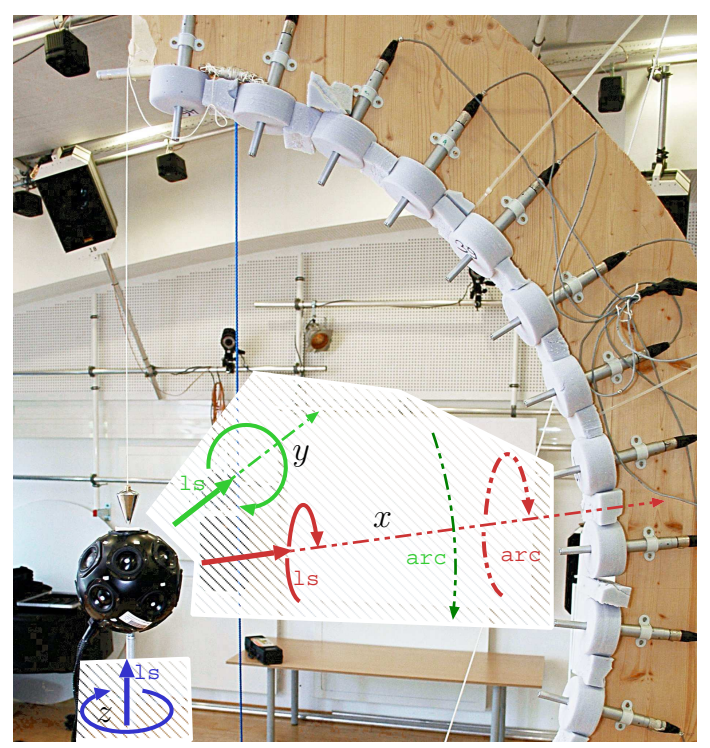

(c) measurement setup

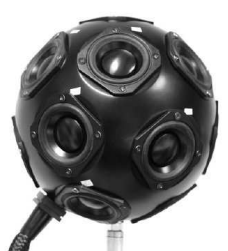

b) mid-frequency array
Fig. 1. Low and mid frequency spherical loudspeaker arrays a), b) that were measured using the microphone array setup in c).

under the displacement $\boldsymbol{d}=[\Delta x, \Delta y, \Delta z]^{\mathrm{T}}$ and rotation

$$
\begin{aligned}
\boldsymbol{R}(\boldsymbol{r}) & :=\boldsymbol{R}_{\mathrm{zy}}^{\mathrm{T}}(\varphi, \vartheta) \boldsymbol{R}_{\mathrm{z}}(r) \boldsymbol{R}_{\mathrm{zy}}(\varphi, \vartheta) \\
\boldsymbol{R}_{\mathrm{zy}}(\varphi, \vartheta) & :=\boldsymbol{R}_{\mathrm{z}}(\varphi)\left[\begin{array}{ccc}
\cos (\vartheta) & 0 & -\sin (\vartheta) \\
0 & 1 & 0 \\
\sin (\vartheta) & 0 & \cos (\vartheta)
\end{array}\right]
\end{aligned}
$$

with the rotation $r$ parametrized in spherical coordinates: $r$ measures the rotation angle and the rotation axis is oriented towards $\boldsymbol{\theta}(\varphi, \vartheta)=[\cos \varphi \sin \vartheta, \sin \varphi \sin \vartheta, \cos \vartheta]^{\mathrm{T}}$, cf. [10].

The spherical loudspeaker array could be shifted with respect to all three coordinate axes by $\boldsymbol{d}_{\mathrm{ls}}=\left[\Delta x_{\mathrm{ls}}, \Delta y_{\mathrm{ls}}, \Delta z_{\mathrm{ls}}\right]$, and rotated by $\boldsymbol{r}_{\mathrm{ls}}$.

The semicircular microphone array could be shifted on the horizontal plane $\boldsymbol{d}_{\mathrm{arc}}=\left[\Delta x_{\mathrm{arc}}, \Delta y_{\mathrm{arc}}, 0\right]$ and rotated around axis on the horizontal plane $\boldsymbol{r}_{\text {arc }}=r_{\text {arc }}\left[\cos \varphi_{\text {arc }}, \sin \varphi_{\text {arc }}, 0\right]$. This covers misalignments of the semicircle axis from the turntable axis and may cause deviations of the virtual microphone array from spherical.

A misplaced semicircular microphone array yields absolute positions $\boldsymbol{X}_{\text {mic }, \mathrm{A}}$ from $\mathbf{X}_{\text {arc }}$, Eq. (6). The more convenient relative positions $\boldsymbol{X}_{\text {mic }}$ are obtained by including the inverse 
loudspeaker misplacement

$$
\begin{aligned}
& \boldsymbol{X}_{\text {mic }}=\boldsymbol{R}\left(\boldsymbol{r}_{\mathrm{ls}}\right)^{\mathrm{T}}\left[\boldsymbol{X}_{\mathrm{mic}, \mathrm{A}}-\boldsymbol{d}_{\mathrm{ls}}\left[\begin{array}{lll}
1 & \ldots & 1
\end{array}\right],\right. \\
& \boldsymbol{X}_{\mathrm{mic}, \mathrm{A}}=\left[\boldsymbol{R}_{\mathrm{z}}\left(0^{\circ}\right) \boldsymbol{X}_{\mathrm{arc}}^{\prime}, \boldsymbol{R}_{\mathrm{z}}\left(10^{\circ}\right) \boldsymbol{X}_{\mathrm{arc}}^{\prime}, \ldots, \boldsymbol{R}_{\mathrm{z}}\left(350^{\circ}\right) \boldsymbol{X}_{\mathrm{arc}}^{\prime}\right] \text {, } \\
& \boldsymbol{X}_{\mathrm{arc}}^{\prime}=\boldsymbol{R}_{\mathrm{z}}\left(\boldsymbol{r}_{\mathrm{arc}}\right) \mathbf{X}_{\mathrm{arc}}+\boldsymbol{d}_{\mathrm{arc}}\left[\begin{array}{lll}
1 & \ldots & 1
\end{array}\right] \text {. }
\end{aligned}
$$

Each column of $\boldsymbol{X}_{\mathrm{mic}}$ corresponds to a microphone measurement position $\boldsymbol{x}_{\text {mic }}^{(i)}$. Geometric errors cause a deviation from the ideal radius $\left\|\boldsymbol{x}_{\text {mic }}^{(i)}\right\| \neq \mathrm{r}_{\mathrm{M}}$ in Eqs. (1) and the ideal directions $\boldsymbol{x}_{\text {mic }}^{(i)} /\left\|\boldsymbol{x}_{\text {mic }}^{(i)}\right\| \neq \boldsymbol{\theta}_{\text {mic }}^{(i)}$ in Eq. (3).

\section{B. Achievable physical alignment}

For positioning the loudspeaker and turntable the following procedure was used: a piece of paper with an $x y$ scale was placed on the top of the loudspeaker at its center. At the $0^{\circ}$ rotation of the turntable, speaker was aligned such that the plumb line hanging from the semicircle microphone array showed $x_{0^{\circ}}=y_{0^{\circ}}=0$ on the $x y$ scale. How much the turntable is eccentric to the plumb line was found after a turntable rotation of $180^{\circ}$, showing $x_{180^{\circ}}=-2 \Delta x_{\text {arc }}$ and $y_{180^{\circ}}=-2 \Delta y_{\text {arc }}$ on the paper scale. Therefore, centering for both loudspeaker and turntable is obtained by shifting both with $-x_{180} / 2$ and $-y_{180} / 2$. To become accurate, the procedure was repeated until the paper scales showed centering errors of only $1 \ldots 2 \mathrm{~mm}$. The height of the loudspeaker was adjusted by the help of a laser pointer, and here the precision is more about in the range of $0.5 \mathrm{~cm}$.

\section{THE EFFECT OF GEOMETRIC ERRORS}

Since the control of spherical loudspeaker arrays is usually done in terms of spherical harmonics, it is reasonable to examine how the different geometric errors affect the spherical harmonic transform of Eq. (3). If no geometric errors are present, then driving the $n, m$ spherical harmonic of the ideal source exclusively yields the $n^{\prime}=n, m^{\prime}=m$ component in $\psi_{n^{\prime} m^{\prime}}=\delta_{n n^{\prime}}^{m m^{\prime}}$ according to Eq. (3). By contrast, misalignments in the measurement setup cause an apparent "crosstalk": an ideal source radiating one spherical harmonic $Y_{n}^{m}$ seems to produce also other components $n^{\prime} \neq n, m^{\prime} \neq m$ in the analysis of the virtual spherical microphone array. This is because ideal angular and radial locations used for the analysis deviate from the real ones.

In general, angular errors result in an apparent frequencyindependent crosstalk between the spherical harmonics through sampling $Y_{n}^{m}(\boldsymbol{\theta})$ at wrong angles. On the other hand, radial (distance) errors result in a frequency-dependent error due to the fact that all the microphone signals are delayed by different amount, which cause phase cancellations when the signals are summed during the spherical harmonic transform. Since the same time delays lead to larger phase differences at high frequencies, distance errors are increasing as a function of frequency.

\section{A. Displacement of the source}

If an ideal source is shifted by $\Delta x_{\mathrm{ls}}, \Delta y_{\mathrm{ls}}$, or $\Delta z_{\mathrm{ls}}$ this will cause both distance and angle errors between the source and

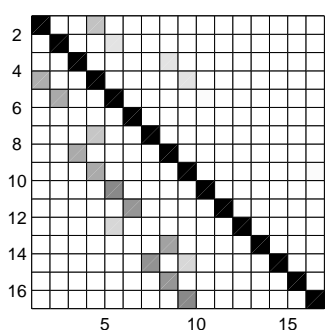

(a) $3 \mathrm{~cm}$ shift along $x$

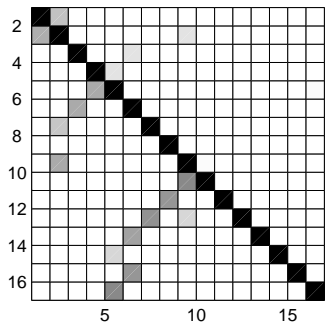

(c) $3 \mathrm{~cm}$ shift along $y$

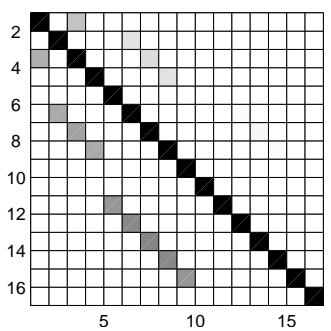

(e) $3 \mathrm{~cm}$ shift along $z$
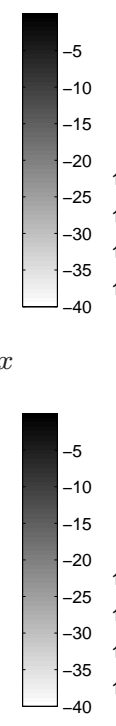

$y$
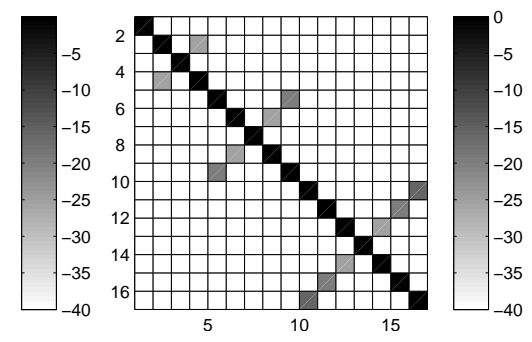

(f) $3^{\circ}$ rotation around $z$
Fig. 2. Spherical harmonic crosstalk in idealized measurement at $1 \mathrm{kHz}$ due to misaligned source in $\mathrm{dB}$. The source is shifted by $3 \mathrm{~cm}$ along $x, y$, or $z$ and rotated by $3^{\circ}$ around $x, y$, or $z$. The indices on the $x$ and $y$ axes correspond to a linear index $q=n^{2}+n+m+1$ used for the spherical harmonics.

observation at the microphone positions. While these errors are generally frequency dependent, here only one frequency $(1 \mathrm{kHz})$ is analyzed. The virtual speaker is an ideal spherical harmonic source described by Eq. (1), whose sound is picked up by a virtual array of microphones with positions that comply with our measurement setup (Sec. II). The simulated microphone signals are encoded to spherical harmonics by Eq. (3). Figure 2 (a), (c), and (e) show how much signal all of the 16 spherical harmonics of the simulated microphone measurement receive when there is a shift along $x, y$, or $z$ of the source. The $q$ th column in each Figure corresponds to an ideal source that radiates one spherical harmonic indexed by $q=n^{2}+n+m+1$. Ideally, the crosstalk matrix should only contain diagonal elements (nonwhite in the Figures).

Plain rotational errors of the source orientation yield a frequency independent leakage between the spherical harmonics as it is displayed in Fig. 2 (b), (d), and (f) for a $3^{\circ}$ rotation around the $x, y$, and $z$ axis, respectively. The crosstalk for rotation can only occur between the spherical harmonics of the same order. 


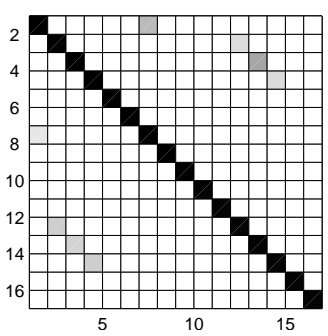

(a) $3 \mathrm{~cm}$ shift along $x$

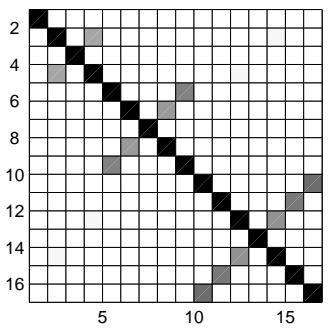

(c) $3 \mathrm{~cm}$ shift along $y$
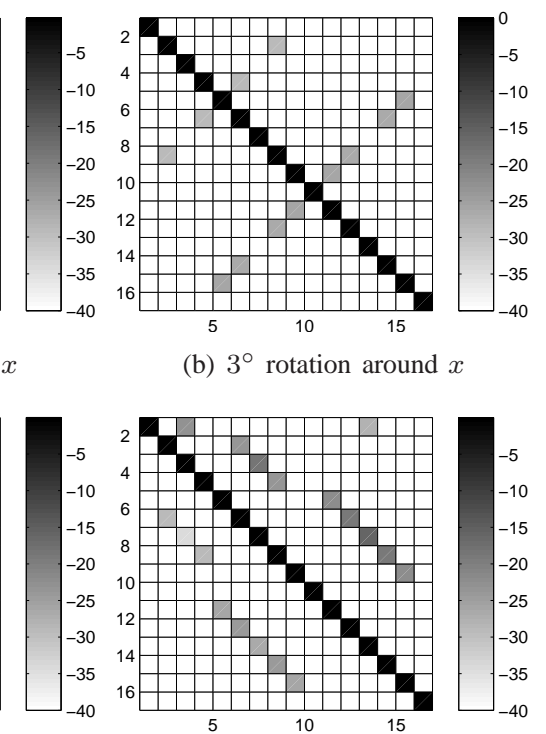

(d) $3^{\circ}$ rotation around $y$
Fig. 3. Spherical harmonic crosstalk in idealized measurement at $1 \mathrm{kHz}$ due to misaligned measurement array. The rotation axis is shifted by $3 \mathrm{~cm}$ along $x$ or $y$, or rotated by $3^{\circ}$ degrees around $x$ or $y$.

\section{B. Displacement of the measurement array}

If the semicircular microphone array is misplaced by $\Delta x_{\text {arc }}$ with regard to the turntable axis, this causes a somewhat ellipsoidal distortion of the virtual microphone array such that its horizontal radius is larger than its vertical one. This mostly yields radial errors for the microphones around the horizontal plane and angular errors for microphones at the top or bottom of the sphere. In between, both effects take place. The crosstalk at $1 \mathrm{kHz}$ is displayed in Fig. 3 (a) for $\Delta x_{\text {arc }}=3 \mathrm{~cm}$.

If the semicircular microphone array is misplaced by $\Delta y_{\text {arc }}$, this will mostly resemble a rotation of the virtual spherical microphone around the $z$-axis. Since there is little distance error, the crosstalk is largely frequency-independent. Fig. 3 (c) displays the crosstalk between spherical harmonics for $\Delta y_{\text {arc }}=3 \mathrm{~cm}$. A displacement of the semicircular array towards $z$ is equivalent to the opposite displacement of the source, which was discussed in Sec. III-A.

If the semicircular array is rotated around the $y$ axis, this is equivalent to shifting the microphone positions with respect to the zenith angle. Therefore, this results in frequency independent angular distortion errors, however with possible crosstalk between the orders. This is shown in Fig. 3 (d). Rotations around $x$ are shown in (b) and are mostly frequency independent, and again with crosstalk between spherical harmonics of different order.

\section{Modeling The Delay of ACOUStiCAL MEASUREMENTS}

The following models use the notation $\mathrm{R}$ for the radius of the loudspeaker array, and $r$ for the radius of an observation point. Loudspeaker positions $\boldsymbol{x}_{\mathrm{ls}}^{(l)}$ enclose polar angles $\vartheta=$ $\arccos <\boldsymbol{x}_{\mathrm{ls}}^{(l)} / \mathrm{R}, \boldsymbol{x}_{\text {mic }}^{(i)} / r>$ with microphone positions $\boldsymbol{x}_{\text {mic }}^{(i)}$.

\section{A. Acoustic model}

The most accurate model of the acoustic delay is the analytic model of sound radiation from a spherical loudspeaker [2], [11]. Fig. 4(a) shows a cross-section of the rotationally symmetric analytic model of a vibrating cap. The normal velocity on the sphere is a unit step function $u(\cdot)$ in the polar angle

$\left.v_{r}\right|_{\mathrm{R}}(\vartheta)=u\left(\frac{\alpha}{2}-\vartheta\right)=\sum_{n=0}^{\infty} \nu_{n} P_{n}(\cos \vartheta)$,

with $\nu_{n}= \begin{cases}1-\cos \frac{\alpha}{2}, & n=0 \\ \frac{2 n+1}{2(n+1)}\left[P_{n-1}\left(\cos \frac{\alpha}{2}\right)-\cos \frac{\alpha}{2} P_{n}\left(\cos \frac{\alpha}{2}\right)\right], & \text { else }\end{cases}$

and $P_{n}$ are the Legendre polynomials, which are angular solutions to rotationally symmetric wave fields [7]. The sound pressure involves spherical Hankel functions and their derivatives, the air density $\rho$, and speed of sound $c$

$$
p(k r, \vartheta)=\frac{\rho c}{\mathrm{i}} \sum_{n=0}^{\infty} \nu_{n} \frac{h_{n}(k r)}{h_{n}^{\prime}(k \mathrm{R})} P_{n}(\cos \vartheta) .
$$

From this, the group delay can be estimated at any point in space.

\section{B. Geometric model}

The geometric model describes the shortest diffraction path from one point on a rigid sphere to a point in space, $\mathrm{cf}$. Fig. 4(b). It has two cases

$$
\hat{\tau}(\vartheta)=\frac{1}{c} \begin{cases}\sqrt{r^{2}+\mathrm{R}^{2}-2 r \mathrm{R} \cos \vartheta}, & r \cos \vartheta-\mathrm{R} \leq 0 \\ \sqrt{r^{2}-\mathrm{R}^{2}}+\left(\vartheta-\arccos \frac{\mathrm{R}}{r}\right) \mathrm{R}, & \text { else. }\end{cases}
$$

A comparison between the frequency independent geometric model Eq. (14) and the acoustical group delay computed from Eq. (13) is plotted in Fig. 5, modeling the mid-frequency array at various angles. It turns out that the acoustical cap model and the geometric model give a perfect match in the estimated group delay when the minimum phase component is removed from the acoustically modeled transfer function (cf. dotted grid lines and dashed lines in Fig. 5).

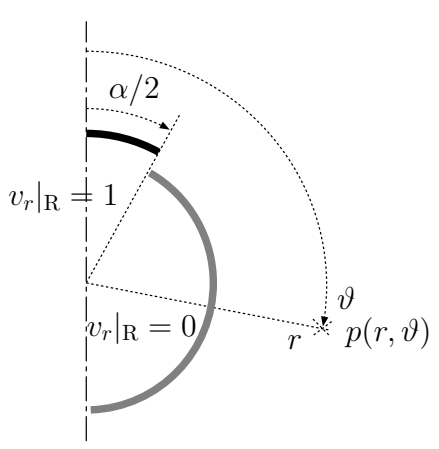

(a) Cap model

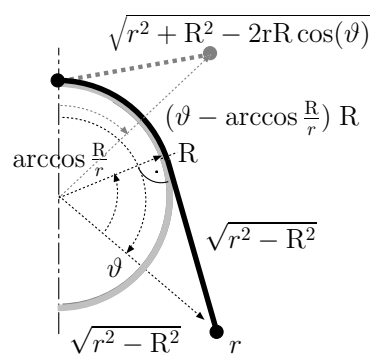

(b) Geom. distance model
Fig. 4. Cap model of sound radiation from spherical loudspeaker and geometric model of acoustic time delays. 


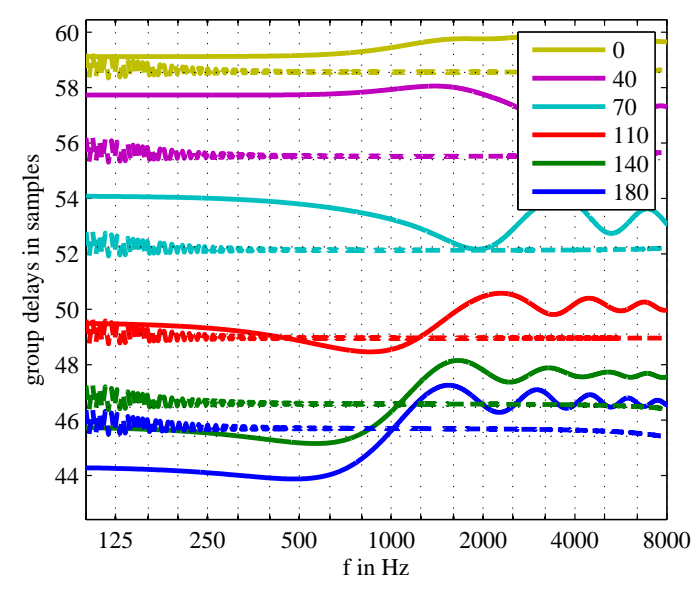

Fig. 5. Comparison of geometric delays (dotted grid lines) and the acoustically modelled group delay with (solid) and without the minimum phase component (dashed) for different angles, and the model of the mid frequency spherical loudspeaker array.

\section{ESTIMATION OF GEOMETRIC ERRORS}

Geometric errors can be estimated by comparing the modeled and measured delays between all loudspeaker elements and microphones.

\section{A. Time-delay estimation}

As we have seen in Secs. IV-A and IV-B, the transfer function from a cap on the sphere to a point in space can be described by a minimum phase transfer function and a delay $H_{\text {rad }}(\omega) e^{-i \omega \tau}$. The transfer function of a loudspeaker $H_{\mathrm{ls}}(\omega)$ and a microphone $H_{\text {mic }}(\omega)$ are usually minimum phase. The whole signal path is

$$
H(\omega)=H_{\mathrm{ls}}(\omega) H_{\mathrm{mic}}(\omega) H_{\mathrm{rad}}(\omega) e^{-\mathrm{i} \omega \tau}=H_{\mathrm{mph}}(\omega) e^{-\mathrm{i} \omega \tau},
$$

where $H_{\mathrm{mph}}(\omega)$ is the minimum phase version of $H(\omega)$ obtained by replacing its phase with the Hilbert transform of the log-magnitude $\ln |H(\omega)|$.

Accordingly, the delay of a particular measurement channel $(i, l)$ is calculated by first removing the minimum phase part

$$
H_{\mathrm{nonmph}}^{(i, l)}(\omega)=\frac{H^{(i, l)}(\omega)}{H_{\mathrm{mph}}^{(i, l)}(\omega)} .
$$

Then, $H_{\text {nonmph }}^{(i, l)}(\omega)$ is converted to the time-domain by inverse the Fourier transform $h_{\text {nonmph }}^{(i, l)}(t)=\operatorname{IFT}\left\{H_{\text {nonmph }}^{(i, l)}(\omega)\right\}$. The delay is found as its peak

$$
\tau^{(i, l)}=\underset{t}{\operatorname{argmax}} h_{\text {nonmph }}^{(i, l)}(t) .
$$

In practice, this is done by IFFT, and the discrete-time signals are upsampled by a factor of 256 prior to peak finding to provide fractional-sample accuracy.

\begin{tabular}{|c|c|c|c|}
\hline sph. array & meas. 1 & meas. 2 & meas. 3 \\
\hline $\boldsymbol{d}_{\mathrm{ls}}$ in $\mathrm{cm}$ & $0.2,0.1,-0.3$ & $0.1,0.3,-0.4$ & $0.2,0.1,-0.3$ \\
\hline $\boldsymbol{r}_{\mathrm{ls}}$ in ${ }^{\circ}$ & $-1.1,-1.2,1.1$ & $-0.6,0.0, \mathbf{6 . 0}$ & $-1.3,-1.4,-0.4$ \\
\hline $\boldsymbol{d}_{\mathrm{arc}}$ in $\mathrm{cm}$ & $0.9,0.7$ & $0.3,0.9$ & $2.8,-1.3$ \\
\hline $\boldsymbol{r}_{\operatorname{arc}}$ in $^{\circ}$ & $0.8,-0.5$ & $0.3,0.9$ & $0.7,-0.7$ \\
\hline
\end{tabular}

TABLE I

GEOMETRIC ERRORS DETECTED IN THREE MEASUREMENTS

\section{B. Geometry estimation with modeled and measured delays}

Rotation and displacement dependent positions of measurement microphones have been modeled in Eq. (11) and they can be employed in Eq. (14) to model time delays $\hat{\tau}^{(i, l)}$ between loudspeakers and microphones. However, rotations and displacements are unknown. Moreover, Eq. (17) has been used to determine time delays from measurements.

The MATLAB Optimization Toolbox function lsqnonlin was used to retrieve the displacements $\left(\boldsymbol{d}_{\mathrm{ls}}, \boldsymbol{d}_{\mathrm{arc}}\right)$ and rotations $\left(\boldsymbol{r}_{\mathrm{ls}}, \boldsymbol{r}_{\mathrm{arc}}\right)$ that are involved in $\hat{\tau}$ by minimizing the mismatch $\hat{\tau}^{(i, l)}-\tau^{(i, l)}$ between model and measurement. The optimizer also retrieved the initial delay $\tau_{0}$ we removed from all impulse response data in common. Delays get inaccurate when the speaker element is on the other side of the sphere, so the mismatch was weighted by $w^{(i, l)}=\cos \left[\min \left(\vartheta^{(i, l)} \frac{180^{\circ}}{150^{\circ}} / 2, \pi / 2\right)\right]$ before minimizing its squared sum

$$
\min \sum_{i, l} w^{(i, l)}\left(\hat{\tau}^{(i, l)}-\tau^{(i, l)}-\tau_{0}\right)^{2} .
$$

The standard deviation of the unweighted mismatch achieves $0.4 \mathrm{~mm}$ for the $36 \times 18 \times \mathrm{L}$ paths after optimization.

\section{Examples}

Table I shows examples that have been evaluated out of selected measurement series done in August 2011 both with the low- and mid-range loudspeaker arrays. Meas. 1 shows the results for an unmodified geometric setup for the midrange array. The estimated total rotation stays below $2^{\circ}$ and the displacement stays below $1 \mathrm{~cm}$. Meas. 2 was done with the icosahedral array which has been rotated on purpose by $5^{\circ}$ around $z$ compared to Meas. 1, leading to an estimated rotation of $6^{\circ}$. Meas. 3 was done with a shifted rotation axis by $\Delta x_{\text {arc }} \approx 2 \mathrm{~cm}$ and $\Delta y_{\text {arc }} \approx-2 \mathrm{~cm}$ compared to meas. 1 , and indeed, $\boldsymbol{d}_{\mathrm{arc}}$ of meas. 3 differs from that of meas. 1 by 1.9 and $-2 \mathrm{~cm}$.

The table indicates that the method yields fairly accurate results with only one or two millimeters of uncertainty for different tests. It is also obvious that the modified parameters are indicated with a quality that is reasonable for monitoring the geometric accuracy.

\section{ERROR COMPENSATION IN MEASURED RESPONSES}

Once the geometric errors are estimated, they can be used in order to correctly interpret the measured responses. The most significant effect of the geometric errors of the measurement setup is that all the microphone signals are delayed differently. A delay compensation is achieved in the frequency domain 

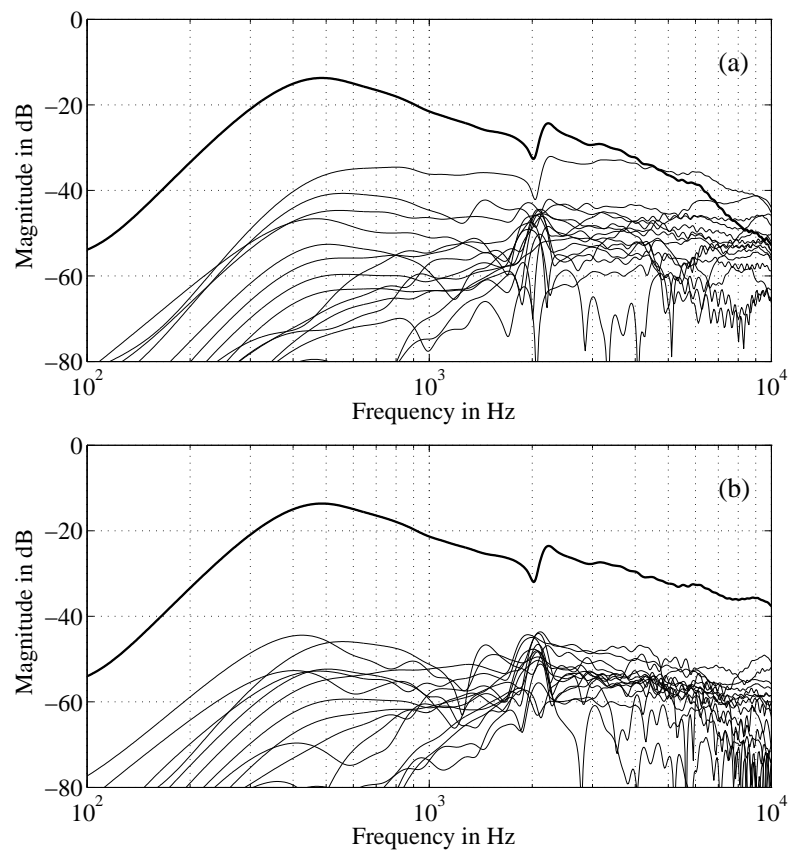

Fig. 6. Measurement of the spherical harmonic responses of the midfrequency array. The loudspeaker array is displaced by $\Delta x_{1 \mathrm{~s}}=2 \mathrm{~cm}$. The first spherical harmonic is excited. The thick line is the response of the first spherical harmonic, the thin lines are harmonics 2-16 which should be zero. In comparison to the uncompensated case (a), the results with geometric error compensation in (b) show significantly less crosstalk.

by applying the compensation $e^{\mathrm{i}(\omega / c)\left(r^{(i)}-\mathrm{r}_{\mathrm{M}}\right)}$. Moreover, the estimated microphone positions have to be used as corrected directions $\boldsymbol{\theta}_{\mathrm{mic}}^{(i)}=\boldsymbol{x}_{\mathrm{mic}}^{(i)} / r^{(i)}$ of the spherical harmonics transform applied in Eq. (3).

Figure 6 displays the spherical harmonic transfer functions of the mid-range loudspeaker array with a $\Delta x_{\mathrm{ls}}=2 \mathrm{~cm}$ displacement. A rough reproduction of spherical harmonic patterns is achieved by superimposing the measured directivities by decoding using $D_{\text {ls }}$ (see Sec. I). In Fig. 6 the first (monopole) harmonic is excited through the decoder. As seen in Fig. 7 (a), which assumes an ideal measurement geometry to calculate $\Psi(\omega)$, not only the first harmonic (thick line) appears at the output, but also the higher spherical harmonics (thin lines). However, when a delay compensation is applied to the microphone signals prior to corrected spherical harmonic decomposition (b), the crosstalk significantly decreases, showing that a large part of the crosstalk was coming from the imperfection of the measurement geometry and not from the loudspeaker array itself. It is clear that when a control system $\tilde{G}(\omega)$ were designed based on the uncorrected measurement of Fig. 6 (a), it would try to correct heavy apparent crosstalk, which would actually worsen the radiation pattern in reality, instead of improving it. Therefore, validating and, if necessary, correcting the radiation measurements of spherical loudspeaker arrays seems to be vital for radiation control.

\section{CONCLUSION}

The radiation pattern of compact spherical loudspeaker arrays is in general controlled based on radiation measurements of the speaker elements. Thus, any error in the measurements will result in a suboptimal control system and an erroneous radiation pattern. In this paper we have demonstrated that geometric measurement errors can introduce significant apparent spherical harmonic crosstalk, even for small misalignments.

We have shown that acoustic delay information can be modeled by a simple geometric model after the non-minimum phase part of the transfer function is removed. Based on this geometric model, an optimization procedure was successfully employed to estimate geometric errors with an accuracy in the range of a millimeter. The geometric estimates were validated by running the optimizer on various data sets where different geometric errors were introduced intentionally.

Moreover, the estimated geometric errors are applied to obtain a substantially corrected interpretation of the measurement data by delay compensation and corrected spherical harmonic transform. More elaborate corrections could take frequency dependent magnitude correction of near field into account in the future, especially for higher order radiation components. Future work also includes applying our method for the measurement of spherical microphone arrays.

\section{ACKNOWLEDGMENT}

This work was supported by the Eötvös Scholarship of the Hungarian Scholarship Board, the Bolyai Scholarship of the Hungarian Academy of Sciences, and by the Hungarian Scientific Research Fund (OTKA), grant nr. TS-73496.

\section{REFERENCES}

[1] O. Warusfel, P. Derogis, and R. Caussé, "Radiation synthesis with digitally controlled loudspeakers," in Papers of the 103rd AES Convention, New York, 1997.

[2] F. Zotter and R. Höldrich, "Modeling radiation synthesis with spherical loudspeaker arrays," in Int. Congress on Acoustics, September, Madrid, 2007.

[3] M. Pollow and G. K. Behler, "Variable directivity for platonic sound sources based on spherical harmonics optimization," Acta Acust. Acust., vol. 95, pp. 1082-1092, 2009.

[4] A. M. Pasqual, P. Herzog, and J. R. F. Arruda, "Theoretical and experimental analysis of the electromechanical behavior of a compact spherical loudspeaker array for directivity control," J. Acoust. Soc. Am., vol. 128 , no. $6,2010$.

[5] I. Witew, G. K. Behler, and M. Vorländer, "About just noticeable differences for aspects of spatial impressions in concert halls," Acoustical Science and Technology, Acoustical Society of Japan, vol. 26, pp. 185-192, 2005. [Online]. Available: http://www.jstage.jst. go.jp/article/ast/26/2/26_185/_article

[6] F. Zotter, "Analysis and synthesis of sound-radiation with spherical arrays," Ph.D. dissertation, University of Music and Performing Arts, Graz, 2009.

[7] E. G. Williams, Fourier Acoustics. London/San Diego: Academic Press, 1999.

[8] F. Zotter, "Sampling strategies for acoustic holography/holophony on the sphere," in NAG-DAGA, Int. Conf., Rotterdam, 2009.

[9] F. Zotter, H. Pomberger, and A. Schmeder, "Efficient directivity pattern control for spherical loudspeaker arrays," in Proceedings of the 2nd ASA-EAA joint conference, ACOUSTICSO8, Paris, Jul 2008.

[10] J. B. Kuipers, Quaternions and Rotation Sequences. Princeton University Press, 2002.

[11] R. M. Aarts and A. J. Janssen, "Comparing sound radiation from a loudspeaker with that from a flexible cap on a rigid sphere," J. Audio Eng. Soc., vol. 59, no. 4, pp. 201-212. 